\title{
Molecular Learning with DNA Kernel Machines
}

\author{
Yung-Kyun Noh ${ }^{\mathrm{a}}$, Daniel D. Lee ${ }^{\mathrm{b}}$, Kyung-Ae Yang ${ }^{\mathrm{c}}$, \\ Cheongtag Kim ${ }^{\mathrm{d}}$, Byoung-Tak Zhang ${ }^{\mathrm{e}}$ \\ ${ }^{a}$ Department of Mechanical and Aerospace Engineering, Seoul National University, Rep. of Korea \\ ${ }^{b}$ Department of Electrical and Systems Engineering, University of Pennsylvania, USA \\ ${ }^{c}$ Department of Medicine, Columbia University, USA \\ ${ }^{d}$ Department of Psychology, Seoul National University, Rep. of Korea \\ ${ }^{e}$ School of Computer Science and Engineering, Seoul National University, Rep. of Korea
}

\begin{abstract}
We present a computational learning method for bio-molecular classification. This method shows how to design biochemical operations both for learning and pattern classification. As opposed to prior work, our molecular algorithm learns generic classes considering the realization in vitro via a sequence of molecular biological operations on sets of DNA examples. Specifically, hybridization between DNA molecules is interpreted as computing the inner product between embedded vectors in a corresponding vector space, and our algorithm performs learning of a binary classifier in this vector space. We analyze the thermodynamic behavior of these learning algorithms, and show simulations on artificial and real datasets as well as demonstrate preliminary wet experimental results using gel electrophoresis.
\end{abstract}

Keywords: DNA computing, machine learning, learning in vitro, kernel methods, molecular algorithms

\section{Introduction}

Molecular computation offers the potential for computing devices to be integrated seamlessly with biological systems [4, 19]. With molecular computation, a machine can be placed inside living tissue to quantitatively measure DNA expression and per5 form pattern recognition in order to identify potential diseases before any physical symptoms emerge. For this purpose, previous research devoted the effort to articulating pattern recognition algorithms to be implemented by biomolecular substrates.

Preprint submitted to Journal of $\mathrm{BT}_{E} X$ Templates

June 23, 2015 
However, most of the previous methods did not consider learning patterns from training examples. For example, a simple implementation of logic gates is proposed for neural networks using metabolic regulation [24], without the implementation of the learning property of neural networks. In [20], solving various dynamic neural network systems is proposed from RNA transcription processes with the light of similarity between two dynamical behaviors, where the realizability of the idea is reported later [21, 55], but the the molecules are designed to perform a pre-determined network.

15 Additionally, [39] proposed a systematic way of constructing feedforward and recurrent neural networks by cascading node units operated by DNA hybridization. In [35] and [27], a weighted sum operation on DNA molecules is designed to realize a simple perceptron algorithm. All these works have demonstrated the utility of molecular computation for solving pattern recognition problems, many of them exploiting the advantage of their inherently parallel interactions. However, these studies were confined to implementing pre-defined perceptrons and did not address the problem of learning. Any of these methods did not try to adapt the computational weight parameters to the pattern of training examples.

In this work, we present a molecular learning algorithm that can perform such pattern recognition in vitro directly on the biological molecules themselves, and provide simulations showing the state-of-the-art recognition accuracy for biological pattern recognition problems. We show how to model DNA sequences as embedded vectors in a vector space and the hybridization operation as a computation of the dot product between these vectors. DNA hybridization can then be interpreted as computing the inner product in the associated feature space via a Mercer kernel, i.e. the well-known kernel trick in machine learning [46]. Using the definition of inner product in a feature space, state-of-the-art pattern recognition algorithms can be used, which are simpler in both understanding and implementing than the conventional artificial neural networks algorithm. In this work, a biomolecular computation process is modeled as a sequence of computing various kernel matrices, resulting in a well-defined learning and classification algorithm.

In our design, each computed kernel element is the pairwise similarity between two DNA molecules. The similarity is measured in vitro through hybridization and can be 
explicitly defined via the interaction energies of complementary DNA base pairs. Our algorithm manipulates populations of DNA sequences via hybridization and denaturing operations, modifying distributions of the associated vectors in the kernel feature space. After learning is performed on data examples, an unknown DNA sequence molecule can be directly classified using the learned weights in the molecular population. Our simulations with biological data show that the proposed algorithm achieves state-ofthe-art performance, comparing favorably with traditional support vector machines and kernel Fisher discriminant analysis algorithms.

We also analyze our algorithm using thermodynamics and kinetics for DNA hybridization. We first obtain the thermodynamic properties for DNA hybridization based on the previous work [22, 43, 45]. DNA thermodynamics explain how the hybridization probability is determined by the change of energy and entropy as well as the temperature. Based on this work, we design the experiment schedule where the learning is performed properly. We provide a simple kinetic model explaining how the kernel matrix can be positive definite for appropriate temperature schedule. The suggested temperature schedule during hybridization is a simple cooling schedule with a constant speed from high temperature to low temperature. We can also apply small variations of the schedule to have different positive definite kernels, where these variations can be compared to the tuning parameters that control the sparsity of kernel matrix.

Specific implementation methods on DNA molecules are also proposed considering the constraint without traditional computing architectures such as semiconductor devices. We designed a small experiment with real DNA molecules and present preliminary experiments demonstrating how our proposed methods can be applied for real in vitro application.

The remainder of the paper is organized as follows: In Section 2, we briefly explain how kernel methods can be used for biological data analysis and in Section 3, we show how DNA sequence data are embedded into a feature space of the associated kernel of hybridized molecules. Section 4 explains the molecular learning and classification algorithm as well as their geometrical interpretation in the associated feature space. In Section 5, we explain how positive definiteness of the kernel can be guaranteed, and in Section 6, we present simulation results on both synthetic and benchmark datasets. In 
Section 7, a discussion on the real implementation is provided, and preliminary in vitro experiment is presented. Finally, we conclude with a discussion in Section 8.

\section{DNA Kernel for Molecular Computation}

Our definition of the kernel measures similarity between DNA sequences using similarities in biomolecular interaction. DNA molecules interact as they diffuse in so-

75 lution through Brownian motion [5], and can generate thermodynamic reactions by binding and unbinding complementary strands until dynamic equilibrium is reached. The resulting quantity of double-stranded sequences reflect biological similarity between DNA sequences, which can be viewed as coefficients of a novel kernel matrix here. We formally define this kernel matrix in this section, and show how it can be used for learning and classification later.

\subsection{Kernel definition using hybridization}

We consider two-class training data composed of $N$ single-stranded DNA molecules. The sequences are the strings from nucleotide alphabets $\Sigma=\{A, T, G, C\}$, and each sequence is labeled with a binary class label $y \in\{+1,-1\}$. Learning occurs on a dataset $\left\{\left|\mathbf{x}_{i}\right\rangle, y_{i}\right\}_{i=1}^{N}$ where $\left|\mathbf{x}_{i}\right\rangle$ is a vector corresponding to the $i$-th sequence, and $y_{i}$ is its label. We also consider the conjugate vector $\left\langle\mathbf{x}_{j}\right|$ of a sequence $j$, and we write a kernel element $K_{i j}$ as the inner product of $i$-th and $j$-th data.

$$
K_{i j}=\left\langle\mathbf{x}_{j} \mid \mathbf{x}_{i}\right\rangle
$$

The embedding of each $\left|\mathbf{x}_{i}\right\rangle$ in a feature space is specified by the kernel matrix $K$, as illustrated in Figure1

In our algorithm, pairwise similarity is defined via the hybridization reaction. Hybridization is the process of binding two single-stranded molecules together to make a double-stranded molecule. For DNA molecules, hybridization affinity exists between nucleotide pairs ' $\mathrm{A}$ ' and ' $\mathrm{T}$ ' and between ' $\mathrm{G}$ ' and ' $\mathrm{C}$ ', which are known to have a complementary relationship. DNA sequences are also directed, distinguished by the 95 5' and 3' ends of sequences. Hybridization occurs by matching the 3' side of one sequence with the 5' end of the other sequence. The complementary version of a DNA 




Figure 1: Each single stranded DNA sequence is mapped into a vector space by an inner product, defined by the amount of resulting double-stranded product in the hybridization reactions.

sequence can be generated by replacing nucleotides according to $(A \rightarrow T, T \rightarrow A, G \rightarrow C$, and $\mathrm{C} \rightarrow \mathrm{G}$ ), and reversing the 5' -3 ' direction. For example, the complementary sequence of [5'-GCCATA-3'] is [5'-TATGGC-3'] as shown in Fig. 2. Complementary pairs are sequences having the greatest hybridization affinity.

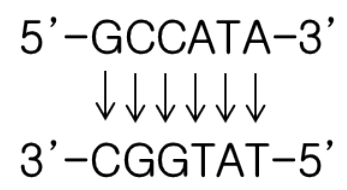

Figure 2: One example of a complementary sequence. Nucleotides ' $\mathrm{A}$ ' and ' $\mathrm{T}$ ', ' $\mathrm{G}$ ' and 'C' are exchanged, and the 5' -3 ' direction is reversed.

Using this notion of complementarity, the definition of a kernel element is straightforward. The kernel element $K_{i j}$ is the quantity of hybridized double strands between sequence $i$ and the complement of sequence $j$ starting from equal amounts of of single- 
stranded sequences, when pairs $i$ and $j$ are mixed:

$$
K_{i j}^{\text {invitro }}=\left|\operatorname{dsDNA}\left(j^{*}, i\right)\right|
$$

Here, we use $j^{*}$ to represent the complementary sequence of $j$. With this definition, the complementary sequence is analogous to the notion of conjugacy when we consider the inner product of $\left|\mathbf{x}_{i}\right\rangle$ and $\left|\mathbf{x}_{j}\right\rangle$. Therefore, we also use the conjugate notation $\langle\mathbf{x}|$ to represent the complementary sequence.

The definition of the DNA kernel in this section, however, does not imply the positive definiteness of the kernel matrix. Reaction dynamics of hybridization are nonlinear and highly complex, and it is difficult to directly control the hybridization process. However, we will show later that if the hybridization procedure is annealed, that is starting from a high temperature and slowly cooling to low temperature, then the resulting kernel matrix will be close to a diagonally dominant positive definite matrix. With this guarantee of positive definiteness, we can design a virtual in vitro algorithm for learning a binary classifier from DNA molecular examples. In the following section, our in vitro learning algorithm is presented along with its geometrical interpretation.

\section{Learning with DNA Kernels}

Here, we show how learning on DNA molecules can be implemented via specially designed biochemical processes. The learning algorithm yields a population of DNA molecules implicitly encoding a weight vector that can be used to classify unknown DNA examples. Our interpretation of the learning algorithm utilizes the Support Vector Machine (SVM) framework [46]. In this context, learning has been considered as finding the closest point within the convex hulls of the class-specific training examples in the feature space [23]. Our in vitro algorithm is similar, but instead learns the convex cones of the training examples, rather than the convex hulls.

We show how convex cones can be learned via populations of DNA molecules encoding kernel matrices using virtual in vitro hybridization processes. These kernel matrices can then be used to readout the class labels of unknown DNA examples again using only in vitro processes. 


\subsection{Learning with hybridization}

We first consider the simplest algorithm, which is to classify an unknown example $\left|\mathbf{x}_{i}\right\rangle$ according to which of two class means is closer [46]. When the two class means are

$$
\vec{c}_{+}=\frac{1}{N_{+1}} \sum_{y_{j}=+1}\left|\mathbf{x}_{j}\right\rangle \quad \text { and } \quad \vec{c}_{-}=\frac{1}{N_{-1}} \sum_{y_{j}=-1}\left|\mathbf{x}_{j}\right\rangle,
$$

\subsection{In vitro processes}

We forge a two-phase process, where in the first phase discriminative information from training examples is learned, and the second phase determines the label of 
an unknown data example. The first in vitro process learns and stores discriminative information in the population of DNA molecules. In this phase, molecules of data examples and complementary sequences are hybridized, yielding two different kinds of double stranded molecules: double strands having differently labeled (hetero-labeled) molecules and double strands having identically labeled (homo-labeled) molecules. Our algorithm iteratively considers the population of hetero-labeled molecules $\left\langle\mathbf{x}_{j} \mid \mathbf{x}_{i}\right\rangle$ for $y_{j} \neq y_{i}$. In this population, the relative frequency of $x_{i}$ molecules are modified according to the ratio between hetero-labeled molecules and all hybridized molecules, given by the quantity $\sum_{y_{j} \neq y_{i}}\left\langle\mathbf{x}_{j} \mid \mathbf{x}_{i}\right\rangle / \sum_{m=1}^{N}\left\langle\mathbf{x}_{m} \mid \mathbf{x}_{i}\right\rangle$. Each step of the learning process results in a population of hetero-labeled double-stranded molecules, which are then denatured, amplified, and used for the next hybridization reaction as shown in Figure 3 . We show that the relative distribution of data molecules $\left|\mathbf{x}_{i}\right\rangle$ in this population reaches a steady-state equilibrium that implicitly stores a set of weight vectors that can be used for classification.

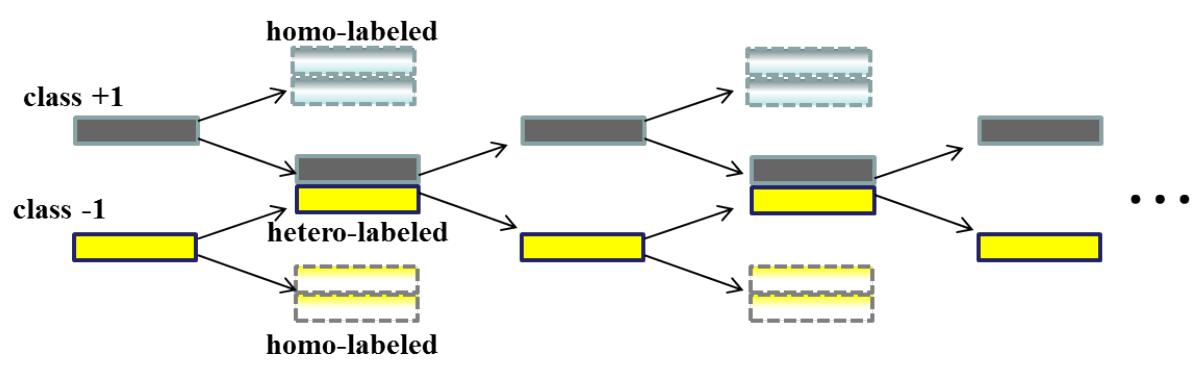

Figure 3: Iterative update of population using hetero-labeled double-stranded molecules.

The classification process uses the learned population from the first phase. An unknown data sequence is hybridized with molecules from this population. The class label of the DNA example is then determined by monitoring the relative amounts of hybridized molecules from each class. Similar to the discrimination algorithm which compared class means, this readout can be performed presumably via an in vitro fluorescence measurement. 


\subsection{Geometrical interpretation}

The proposed learning process can be interpreted geometrically as finding a weight vector in the DNA feature space that is normal to the classification boundary. This weight vector is the difference between the two closest vectors $\mathbf{w}_{\{+1\}}$ and $\mathbf{w}_{\{-1\}}$ that are contained in the convex cones of the two classes as shown in Fig. 4 . Here we prove that the proposed learning method converges to a population of DNA molecules representing this difference of the two closest vectors. The discrimination phase then classifies unknown DNA molecules by taking the inner product with this difference vector.

We represent the weight vector as $\mathbf{w}=\sum_{i=1}^{N} \alpha_{i}\left|\mathbf{x}_{i}\right\rangle$, where $\alpha_{i}$ is the relative concentration of sequence $i$ compared to the initial population, and $\left|\mathbf{x}_{i}\right\rangle$ represents each datum vector in the feature space. The initial $\alpha_{i} \mathrm{~s}$ are uniformly set to one, and a column vector $\alpha=\left\{\alpha_{1}, \ldots, \alpha_{N}\right\}^{T}$ is used to represent the population concentrations at a particular time.

Without loss of generality, the data are sorted with respect to their labels $y_{l} \in$ $\{+1,-1\}, l=1, \ldots, N$, and we separate $\alpha \in \mathbb{R}^{N}$ into two vectors $\alpha_{\{\mathbf{c}\}} \in \mathbb{R}^{N_{c}}$, for class $c \in\{+1,-1\}$ and $K \in \mathbb{R}^{N \times N}$ into four matrices $K_{\left\{\mathbf{c}_{1}, \mathbf{c}_{2}\right\}} \in \mathbb{R}^{N_{c_{1}}, N_{c_{2}}, c_{1}, c_{2}} \in$ $\{+1,-1\}$, where $N_{c}$ is the number of data of class $c$, which satisfies $N_{c=+1}+N_{c=-1}=$ $N$. The generation of double stranded DNA is bilinear in the number of single stranded components $\alpha_{i}$ and $\alpha_{j}$, so that $\left|\operatorname{dsDNA}\left(j^{*}, i\right)\right|=\alpha_{i} \alpha_{j} K_{i j}$. The following theorem shows how the concentrations converge by iteratively hybridizing and amplifying the hetero-labeled double stranded DNA molecules.

Theorem 1: If the selection of hetero-labeled molecules from step $t$ to $t+1$ satisfies the following equation,

$$
\alpha_{i}^{t+1}=\alpha_{i}^{t}\left(\sum_{y_{j} \neq y_{i}} K_{i j} \alpha_{i}^{t} \alpha_{j}^{t} / \sum_{m=1}^{N} K_{i m} \alpha_{i}^{t} \alpha_{m}^{t}\right)=\alpha_{i}^{t}\left(\sum_{y_{j} \neq y_{i}} K_{i j} \alpha_{j}^{t} / \sum_{m=1}^{N} K_{i m} \alpha_{m}^{t}\right)
$$

for $i \in\{1, \ldots, N\}$, the asymptotic distribution of $\alpha=\left\{\alpha_{1}, \ldots, \alpha_{N}\right\}^{T}$ optimizes the following objective function:

$$
\begin{aligned}
& \max _{\alpha_{\{\mathbf{i}\}} \in \mathbb{R}^{N_{i}, i=+1,-1}} \frac{\mathbf{w}_{\{-\mathbf{1}\}}{ }^{T} \mathbf{w}_{\{+\mathbf{1}\}}}{\left\|\mathbf{w}_{\{-\mathbf{1}\}}\right\|\left\|\mathbf{w}_{\{+\mathbf{1}\}}\right\|} \\
& \text { s.t. } \quad \mathbf{w}_{\{-\mathbf{1}\}}=\left\langle\mathbf{x}_{\{-\mathbf{1}\}}\right| \alpha_{\{-\mathbf{1}\}}, \quad \mathbf{w}_{\{+\mathbf{1}\}}=\left\langle\mathbf{x}_{\{+\mathbf{1}\}}\right| \alpha_{\{+\mathbf{1}\}}, \quad \text { for } \quad \alpha_{\{-\mathbf{1}\}}, \alpha_{\{+\mathbf{1}\}} \geq 0 .
\end{aligned}
$$



ized eigenvalue problem [57]:

$$
\left(\begin{array}{cc}
0 & K_{\{-\mathbf{1},+\mathbf{1}\}} \\
K_{\{+\mathbf{1},-\mathbf{1}\}} & 0
\end{array}\right) \alpha=\lambda\left(\begin{array}{cc}
K_{\{-\mathbf{1},-\mathbf{1}\}} & 0 \\
0 & K_{\{+\mathbf{1},+\mathbf{1}\}}
\end{array}\right) \alpha
$$

Here, $\alpha=\left[\alpha_{\{-\mathbf{1}\}}^{T} \alpha_{\{+\mathbf{1}\}}^{T}\right]^{T}$. To show the equivalence between Eqs. 7$\}$ and $[8$, we first reformulate Eq. (7) as maximization of the objective function $L$ with Lagrange multipliers $\lambda_{\{+1\}}$ and $\lambda_{\{-1\}}$ :

$$
\begin{aligned}
L= & \mathbf{w}_{\{-\mathbf{1}\}}{ }^{T} \mathbf{w}_{\{+\mathbf{1}\}}-\frac{1}{2} \lambda_{\{-1\}}\left(1-\mathbf{w}_{\{-\mathbf{1}\}}{ }^{T} \mathbf{w}_{\{-\mathbf{1}\}}\right)-\frac{1}{2} \lambda_{\{+1\}}\left(1-\mathbf{w}_{\{+\mathbf{1}\}}{ }^{T} \mathbf{w}_{\{+\mathbf{1}\}}\right) \\
= & \alpha_{\{-\mathbf{1}\}}{ }^{T}\left\langle\mathbf{x}_{\{-\mathbf{1}\}} \mid \mathbf{x}_{\{+\mathbf{1}\}}\right\rangle \alpha_{\{+\mathbf{1}\}}-\frac{1}{2} \lambda_{\{-1\}}\left(1-\alpha_{\{-\mathbf{1}\}}\left\langle^{T} \mathbf{x}_{\{-\mathbf{1}\}} \mid \mathbf{x}_{\{-\mathbf{1}\}}\right\rangle \alpha_{\{-\mathbf{1}\}}\right) \\
& -\frac{1}{2} \lambda_{\{+1\}}\left(1-\alpha_{\{+\mathbf{1}\}}{ }^{T}\left\langle\mathbf{x}_{\{+\mathbf{1}\}} \mid \mathbf{x}_{\{+\mathbf{1}\}}\right\rangle \alpha_{\{+\mathbf{1}\}}\right) \\
= & \alpha_{\{-\mathbf{1}\}}{ }^{T} K_{\{-\mathbf{1},+\mathbf{1}\}} \alpha_{\{+\mathbf{1}\}}-\frac{1}{2} \lambda_{\{-1\}}\left(1-\alpha_{\{-\mathbf{1}\}}{ }^{T} K_{\{-\mathbf{1},-\mathbf{1}\}} \alpha_{\{-\mathbf{1}\}}\right) \\
& -\frac{1}{2} \lambda_{\{+1\}}\left(1-\alpha_{\{+\mathbf{1}\}}{ }^{T} K_{\{+\mathbf{1},+\mathbf{1}\}} \alpha_{\{+\mathbf{1}\}}\right)
\end{aligned}
$$

The derivatives of $L$ with respect to $\alpha_{\{-\mathbf{1}\}}$ and $\alpha_{\{+\mathbf{1}\}}$ are zero at the maximal points:

$$
\begin{aligned}
& \left(\frac{\partial L}{\partial \alpha_{\{-\mathbf{1}\}}}\right)^{T}=K_{\{-\mathbf{1},+\mathbf{1}\}} \alpha_{\{+\mathbf{1}\}}-\lambda_{\{-1\}} K_{\{-\mathbf{1},-\mathbf{1}\}} \alpha_{\{-\mathbf{1}\}}=0 \\
& \left(\frac{\partial L}{\partial \alpha_{\{+\mathbf{1}\}}}\right)^{T}=K_{\{+\mathbf{1},-\mathbf{1}\}} \alpha_{\{-\mathbf{1}\}}-\lambda_{\{+1\}} K_{\{+\mathbf{1},+\mathbf{1}\}} \alpha_{\{+\mathbf{1}\}}=0
\end{aligned}
$$

If we compare Equations $\sqrt{10}$ and $\sqrt{11}$ after multiplying $\alpha_{\{-1\}}{ }^{T}$ to the left of $[10\}$ and $\alpha_{\{+\mathbf{1}\}}^{T}$ to the left of 11, , we see $\lambda_{\{-1\}}=\lambda_{\{+1\}}$ because we constrained $\mathbf{w}_{\{+1\}}{ }^{T} \mathbf{w}_{\{+\mathbf{1}\}}=$ $\mathbf{w}_{\{-\mathbf{1}\}}{ }^{T} \mathbf{w}_{\{-1\}}=1$. Eq. 8 becomes equivalent to 10 and $(11$, , yielding the result $\lambda=\lambda_{\{-1\}}=\lambda_{\{+1\}}=\mathbf{w}_{\{-\mathbf{1}\}}{ }^{T} \mathbf{w}_{\{+\mathbf{1}\}}$. Thus, the principal eigenvalue $\lambda$ becomes the maximum value of $\mathbf{w}_{\{-\mathbf{1}\}}{ }^{T} \mathbf{w}_{\{+\mathbf{1}\}}$, completing the proof. 
We consider the matrix $\left|\mathbf{x}_{\{\mathbf{c}\}}\right\rangle$ in Theorem 1 as the collection of column vectors $\left|\mathbf{x}_{i}\right\rangle$ in class $c$. Then, the optimized vectors $\mathbf{w}_{\{-\mathbf{1}\}}$ and $\mathbf{w}_{\{+\mathbf{1}\}}$, become the closest vectors where $\mathbf{w}_{\{-\mathbf{1}\}}$ is contained within the positive cone of class -1 , and $\mathbf{w}_{\{+\mathbf{1}\}}$ is contained within the positive cone of class +1 as shown in Fig. 4 .

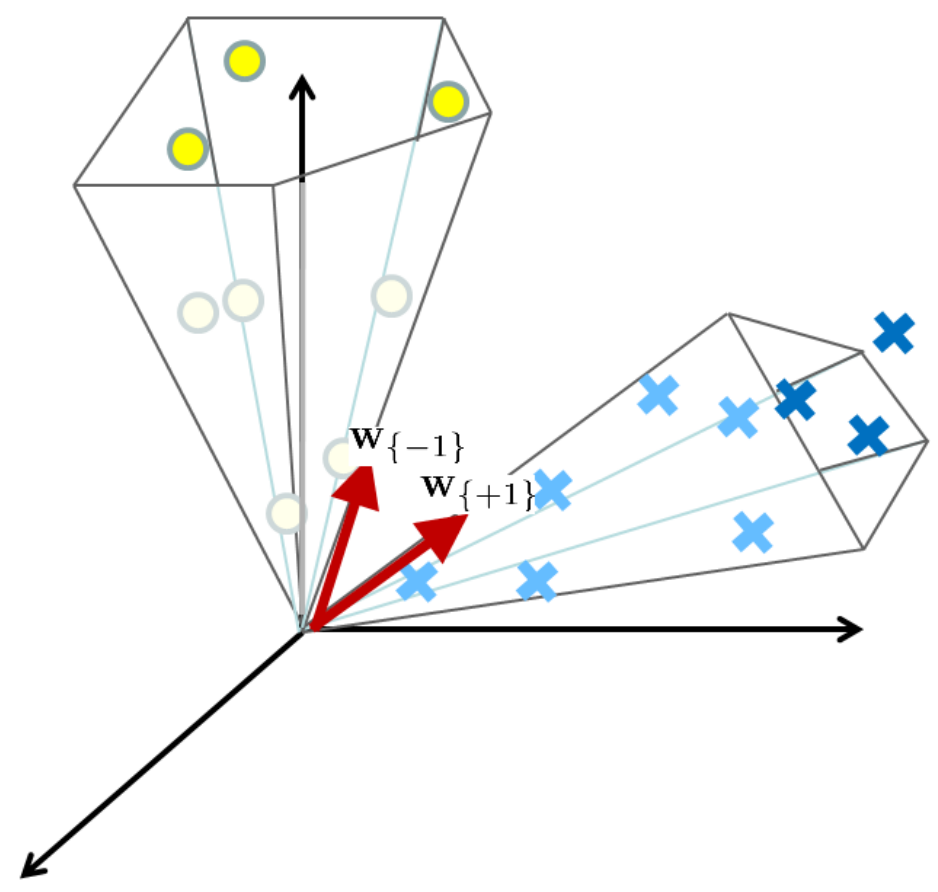

Figure 4: Iterative updates of the hetero-labeled DNA molecules finds the two closest vectors in the feature space which are contained in convex cones of different classes.

The discriminative in vitro phase is then interpreted as determining whether a new test datum $\left|\mathbf{x}_{n e w}\right\rangle$ is closer to $\mathbf{w}_{\{-1\}}$ or $\mathbf{w}_{\{+\mathbf{1}\}}$ in angle. The new DNA molecules are first hybridized with the learned distribution of molecules, resulting in double stranded DNA concentrations given by $\alpha_{i} K(n e w, i)$. Comparison of the amounts of hybridized molecules is equivalent to determining whether $\sum_{y_{i}=+1} \alpha_{i} K($ new, $i)=$ $225 \mathbf{w}_{\{+1\}}^{T}\left|\mathbf{x}_{\text {new }}\right\rangle$ or $\sum_{y_{i}=-1} \alpha_{i} K($ new,$i)=\mathbf{w}_{\{-\mathbf{1}\}}^{T}\left|\mathbf{x}_{\text {new }}\right\rangle$ is larger. This can be viewed as computing the inner product of the new test example with the vectors $\mathbf{w}_{\{+\mathbf{1}\}}$ 
and $\mathrm{w}_{\{-1\}}$, giving the binary class label:

$y_{\text {new }}=\operatorname{sign}\left\{\sum_{i} \alpha_{i} y_{i} K(\right.$ new,$\left.i)\right\}=\operatorname{sign}\left\{\mathbf{w}_{\{+\mathbf{1}\}}^{T}\left|\mathbf{x}_{\text {new }}\right\rangle-\mathbf{w}_{\{-\mathbf{1}\}}^{T}\left|\mathbf{x}_{\text {new }}\right\rangle\right\}$.

The classification decision boundary is given by an hyperplane orthogonal to the difference vector $\mathbf{w}_{\{+\mathbf{1}\}}-\mathbf{w}_{\{-1\}}$.

We have explained the learning and classification process on DNA molecules using simple in vitro operations. In the following section, we verify that positive definite kernel matrices can be properly formed using thermodynamic models of hybridization.

\section{Hybridization Kernel and Positive Definiteness}

When hybridized results are regarded as kernel elements for classification, a positive definiteness [46] or at least a near-positive definiteness [15, 37] of the resulting matrix should be achieved. In this section, we discuss the positive definiteness of the proposed kernel using a kinetic model of hybridization with thermodynamics incorporated. The kinetic model provides predictions for how the different kernel matrix coefficients change as a function of temperature, and we show how an appropriate positive definite kernel matrix can be formed under an annealed temperature schedule.

We consider hybridization between a primary sequence molecule $\left|\mathbf{x}_{i}\right\rangle$ and a complementary molecule $\left\langle\mathbf{x}_{j}\right|$. When $\left|\mathbf{x}_{i}\right\rangle$ and $\left\langle\mathbf{x}_{j}\right|$ hybridize to make a double strand, the process is considered to be a stochastic process of continuous hybridization and denature with transition probabilities $p_{h}$ and $p_{d}$. These transition probabilities are governed by the hybridization energy and entropy changes, written as $\Delta E$ (kcal) and $\Delta S$ $(\mathrm{kcal} / \mathrm{K})$ respectively, as well as by the temperature $T(\mathrm{~K})$, and we can understand hybridization a Monte Carlo Markov Chain (MCMC) process with the transition probabilities [22],

$$
\begin{aligned}
& p_{h}=\left\{\begin{array}{cc}
\exp \left(-G / c_{k} T\right), & G \equiv \Delta E-T \Delta S \geq 0 \\
1, & G \equiv \Delta E-T \Delta S<0
\end{array}\right. \\
& p_{d}=\left\{\begin{array}{cc}
1, & G \equiv \Delta E-T \Delta S \geq 0 \\
\exp \left(G / c_{k} T\right), & G \equiv \Delta E-T \Delta S<0
\end{array}\right.
\end{aligned}
$$


When two particular single stranded molecules of the same amount hybridize, this

MCMC process will make a Boltzmann distribution at equilibrium: the ratio between the concentration of double strands and the square of single strands will be proportional to $\exp \left\{-(\Delta E-T \Delta S) / c_{k} T\right\}$ with a Boltzmann constant $c_{k}=3.2982 \times 10^{4}(\mathrm{kcal} / \mathrm{K})$. However, in a modeling of complex hybridization with many different molecules, the final distribution does not follow the Boltzmann distribution but stays in a different equilibrium. In this situation, the kinetics of hybridization are more important for explaining the population at equilibrium.

Previously, [6] found that the amount of hybridization is not primarily governed by the thermodynamics but follows the similar kinetics regardless of the kinds of binding, once the temperature is below a certain level. This is because the frequency of collision becomes more important than the binding probability itself, if the binding probability is high enough. In their analysis on hybridization, it is claimed that the population of single stranded molecules $C$ changes over time $t$ when the initial concentration of single stranded molecules is $C_{0}$ :

$$
\frac{C}{C_{0}}=\frac{1}{1+\alpha C_{0} t} .
$$

Here, the estimation results showed that the constant $\alpha$ is about the same over various DNAs. Now, this equation can be approximated with small $t$ considering that the majority of the population change occurs at the early stage when $C$ is high:

$$
C \approx C_{0}-\alpha C_{0}^{2} t
$$

From this equation, we can see that the amount of hybridized molecules is approximately proportional to the hybridization time and the square of the initial concentration of single stranded molecules, while the hybridization speed is not governed by the hybridization energy or entropy. Therefore, we can make a hybridization model where the amount of hybridization is proportional to the time of hybridization, when the temperature is within a regime where hybridization can occur. From Eq. (13), we assume that the regime is the temperature below $T_{\theta} \equiv \Delta E / \Delta S$.

Now, we consider hybridization with a cool-down schedule from a high temperature $T_{i}$ to a low temperature $T_{f}$ with a constant speed. Once the temperature passes 
the threshold temperature $T_{\theta} \equiv \Delta E / \Delta S$, the hybridization begins. Upon these settings, the amount of hybridized molecules can be approximated as proportional to the following terms:

$$
\left\langle\mathbf{x}_{j} \mid \mathbf{x}_{i}\right\rangle \propto\left[T_{\theta}(i, j)-T_{f}\right]_{+}-\left[T_{\theta}(i, j)-T_{i}\right]_{+}
$$

Here $[\cdot]_{+}=\max [0, \cdot]$ is the rectification nonlinearity and the threshold temperature binding energy $\Delta E_{i j}$ and entropy change $\Delta S$.

Eq. (16) can be interpreted to mean that the speed of accumulation of double strands is assumed to be zero when the current temperature is higher than the threshold temperature $T_{\theta}$, and the speed is constant when the temperature is lower than $T_{\theta}$. In this matrix.

Because the kernel element is a function of the threshold temperature for binding $T_{\theta}$, we can represent the kernel elements using the binding energies. For simplicity of the analysis, we use a previous work showing that the hybridization energy and entropy 

binding energies are between $A$ and $T$, or $\Delta E_{A T}$, between $C$ and $G$, or $\Delta E_{C G}$, and the binding energy for other pairs, or $\Delta E_{\text {Oth }}$ [22]. Table 1 shows the experimentally determined values for these binding energies.

Table 1: Binding energy of the individual pairs (Kcal/mole base pair (MBP)).

\begin{tabular}{|l|l|}
\hline C-G \& G-C & $\Delta E_{C G}=-9.0$ \\
\hline A-T \& T-A & $\Delta E_{A T}=-7.2$ \\
\hline A-G \& G-A & \\
A-C \& C-A & $\Delta E_{O t h}=-5.3$ \\
T-G \& G-T & \\
T-C \& C-T & \\
\hline
\end{tabular}

Using these binding energies, we show that a sufficient condition for the matrix $K$ to be positive definite. In the following theorem, we consider hybridization of DNA molecules of length $l$, where all sequences share a common set of $m$ nucleotides.

Theorem 2: For sequences of length l, having length $r$ of variable nucleotides and length $m$ of a common nucleotide fragment $(l=r+m)$, and when common nucleotides have an average binding energy of $\Delta E_{g}$, the matrix $K$ from Eq. (16) is positive semi-definite if $T_{i}$ and $T_{f}$ satisfy $T_{i}>-\left(r \Delta E_{C G}+m \Delta E_{g}\right) / \Delta S$ and $T_{f}<-\left(r \Delta E_{O t h}+m \Delta E_{g}\right) / \Delta S$.

Proof: We prove the positive semi-definiteness of matrix $K$ by proving the positive semi-definiteness of each matrix from the two $[\cdot]_{+} \mathrm{s}$ in Eq. 16 separately. First, the term $T_{\theta}-T_{f}$ can be represented as

$$
\frac{1}{\Delta S}\left\{-\Delta E_{i j}^{r}+r \Delta E_{O t h}-r \Delta E_{O t h}-m \Delta E_{g}-T_{f} \Delta S\right\}
$$

where $\Delta E_{i j}^{r}$ is the binding energy of varying sequences of length $r$ satisfying $\Delta E_{i j}=$ $\Delta E_{i j}^{r}+m \Delta E_{g}$. We see that the matrix $-\Delta E^{r}+r \Delta E_{O t h} \mathbf{1}_{N} \mathbf{1}_{N}^{T}$, where each element 
is the first two terms in (17), is positive definite. Here, $\mathbf{1}_{N}$ is a column vector whose $N$ elements are all unitary. Consequently, $-\frac{\Delta E}{\Delta S}-T_{f} \mathbf{1}_{N} \mathbf{1}_{N}^{T}$ is positive definite when the other terms in $(17),-\left(r \Delta E_{O t h}+m \Delta E_{g}+T_{f} \Delta S\right)$ are positive, which gives the condition $T_{f}: T_{f}<-\left(r \Delta E_{O t h}+m \Delta E_{g}\right) / \Delta S$. Second, if $-\frac{\Delta E_{i j}}{\Delta S}-T_{i}$ in the second $[\cdot]_{+}$term are all negative, then the matrix remains positive definite. This yields the additional condition $T_{i}>-\left(r \Delta E_{C G}+m \Delta E_{g}\right) / \Delta S$.

Theorem 2 shows that positive definiteness of the kernel matrix is ensured if it is annealed from a temperature higher than $-\frac{r \Delta E_{C G}+m \Delta E_{g}}{\Delta S}$ to a temperature lower than $-\frac{r \Delta E_{O t h}+m \Delta E_{g}}{\Delta S}$. These bounds are also controlled by the average binding energy $\Delta E_{g}$ of a common nucleotide fragment. This value $\Delta E_{g}$ is always between $\Delta E_{C G}$ and $\Delta E_{A T}$, and the presence of common nucleotides always decreases the lower bound on the initial temperature $T_{i}$ and increases the upper bound on the final temperature $T_{f}$.

For example, if we consider molecular sequences of length $l=100$, and if they have common nucleotide fragments of 50 ' $A$ 's and 20 ' $T$ 's, then $\Delta E_{g}=-7.79(\mathrm{kcal})$ according to Table 1 , and $m=70$ and $r=30$. We also use $\Delta S=c_{s} l$ where $c_{s}=$ $0.023(\mathrm{kcal} / \mathrm{K})$ for the entropy calculation [22]. In this case, according to Theorem 2, the kernel satisfies positive definiteness if $T_{i}>(9 \times 30+7.79 \times 70) /(0.023 \times 10)(K)=$ $81.33\left({ }^{\circ} \mathrm{C}\right)$ and $T_{f}<33.07\left({ }^{\circ} \mathrm{C}\right)$, which is easily satified during the experimental DNA hybridization process.

More complex DNA binding models relax the assumption of independent binding energies per base pair. For example, a context-dependent binding method can be used which considers consecutive pairs of neighborhood sites [44]. Even with these more complex models, analytic calculations show that the in vitro DNA kernel matrix will remain positive definite if the proper annealing schedule is followed.

\section{Experimental Results}

In this section, we present several simulation experiments to show how the proposed algorithm can perform classification tasks. In the simulation, we first see that positive definiteness can be achieved by using the appropriate temperature schedule. 
perature schedules. Second, we simulate our learning algorithm with real biological datasets and compare the results against standard machine learning algorithms. The performance of the proposed algorithm is compared to the well-known kernel classification algorithms, SVMs and kernel Fisher discriminant analysis (kFDA), using the mentary sequence $j$, as well as the temperature.

The system from Eq. (18) is nonlinear, where the population distribution can arrive more than one stationary distribution. Because $k_{d}$ and $k_{h}$ are functions of the temperature, the final distribution of single and double stranded molecules is determined 375 six sequences and their complementary sequences and tested how annealing process, 

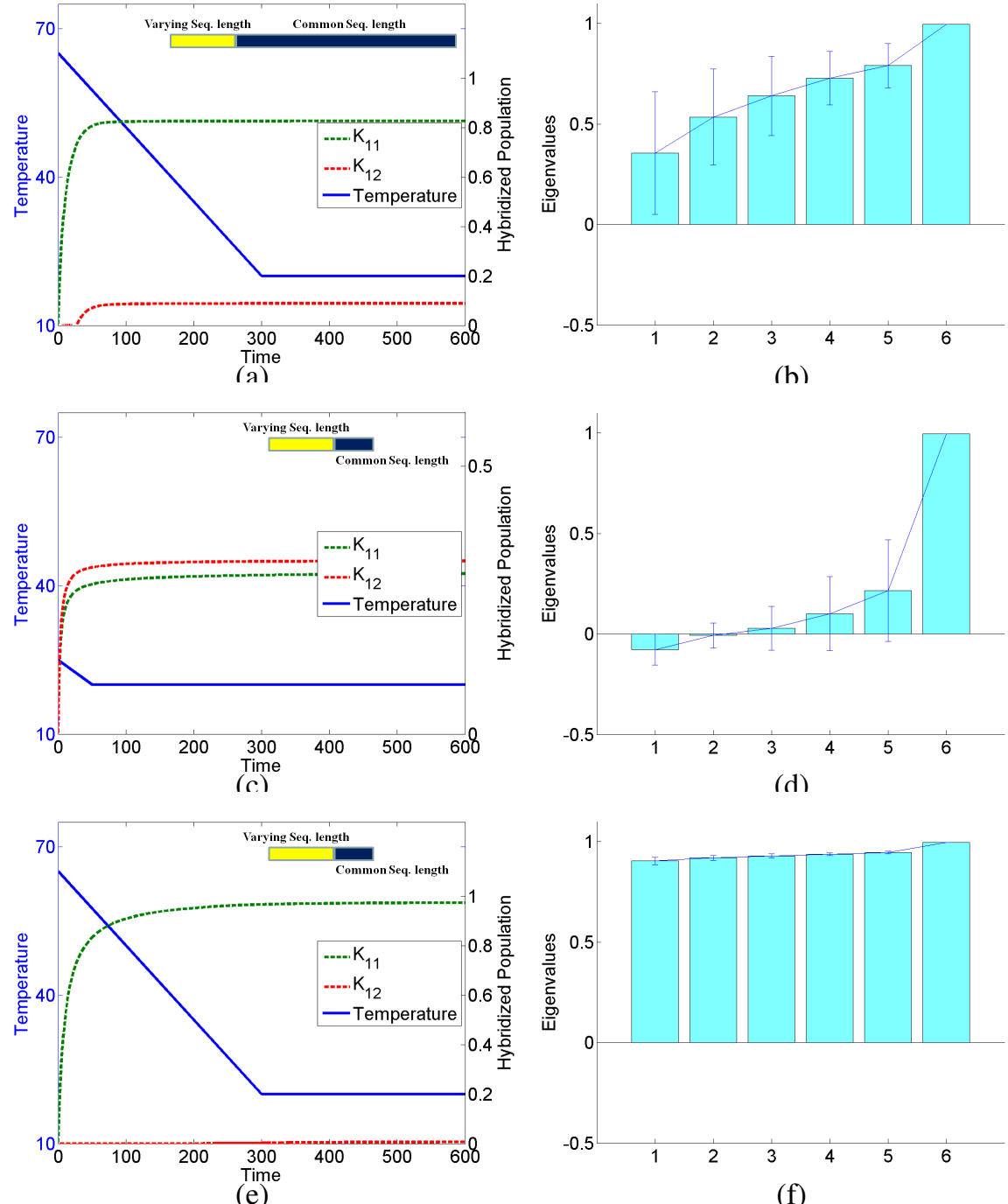

Figure 5: Simulation of kernel values. Six random primary sequences are hybridized together with their complementary sequences using Eq. 18]. The primary sequences include a common sequence where the relative length is also represented. In (a), (c), and (e), the temperature schedules and the resulting $K_{11}$ and $K_{12}$ are plotted over time for one example of random sequence. In (b), (d), and (f), the mean and the covariance of 6 eigenvalues of generated kernels are shown for experiments in (a), (c), and (e), respectively. In (c) and (d), annealing is not sufficient, and in (e) and (f), less common sequence is used than (a) and (b).

proposed in Section 4, helps generate a positive definite kernel. In Fig. 5, two differ- 
ent annealing schedules are used in Fig. 5(a) and (c), and the construction curves of one diagonal $\left(K_{11}\right)$ element and one off-diagonal $\left(K_{12}\right)$ element are presented. When the annealing started from a higher temperature as in Fig. 5(a), the difference between the diagonal and off-diagonal elements is much larger than the annealing in Fig. 55(c), producing diagonal dominant kernel matrices. In this case, the matrix tends to be positive definite as shown in Fig. 5 (b), which can be compared with the result with non-sufficient annealing in Fig. 5(d) representing a non-positive definite matrix.

In Fig. 5(e) and (f), temperature schedule is the same as the experiments in Fig.5.a) and (b), while the less length of common sequence is used. While the matrix is positive definite in this case, the off-diagonal elements become too sparse as in Fig. 5(e), producing a non-informative kernel matrix, where every pair of data are orthogonal in the feature space.

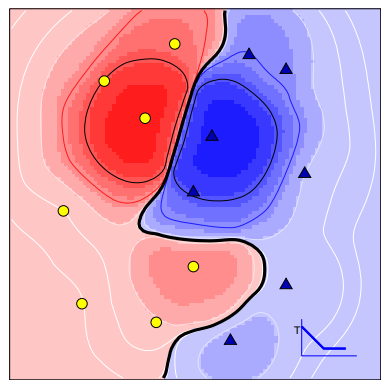

(a)

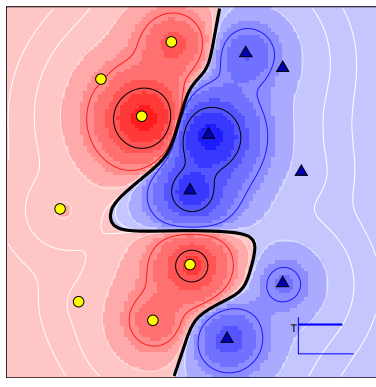

(b)

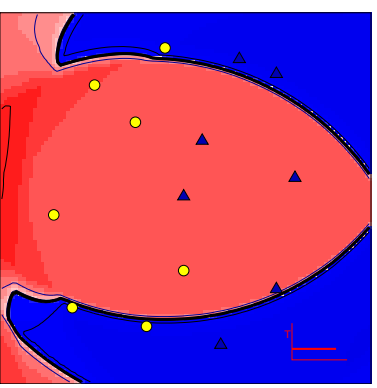

(c)

Figure 6: Two-class data are distributed in a two dimensional space and labeled as yellow and blue. Binding energy between pairs of points are determined by the Euclidean distance and scaled so that the energy is within the range of $-56 \sim-84(\mathrm{kcal})$. The length of binding nucleotides is set to $l=10$ for the entropy calculation. The data are learned with different temperature schedules; test results across the data space is presented as red and blue for the yellow and blue classes respectively. Temperatures for hybridization is (a) $80^{\circ} \mathrm{C}$ to $20^{\circ} \mathrm{C}$, (b) $80^{\circ} \mathrm{C}$ constant, and (c) $30^{\circ} \mathrm{C}$ constant. The kernel in (c) does not satisfy positive definiteness.

Once a positive definiteness can be achieved by annealing, a sparseness of the kernel can be further controlled by modifying the temperature schedule. Conventional kernels tune their sparsity using kernel parameters, such as the width parameter in a Gaussian kernel. In our proposed kernel, we can use the temperature schedule to tune 
the kernel as the width parameter does. For example, a sparse matrix can be generinformation between expression levels and labels. Each gene expression value is simplified by binary thresholding, and are encoded as 'A' and ' $G$ ' respectively for our 


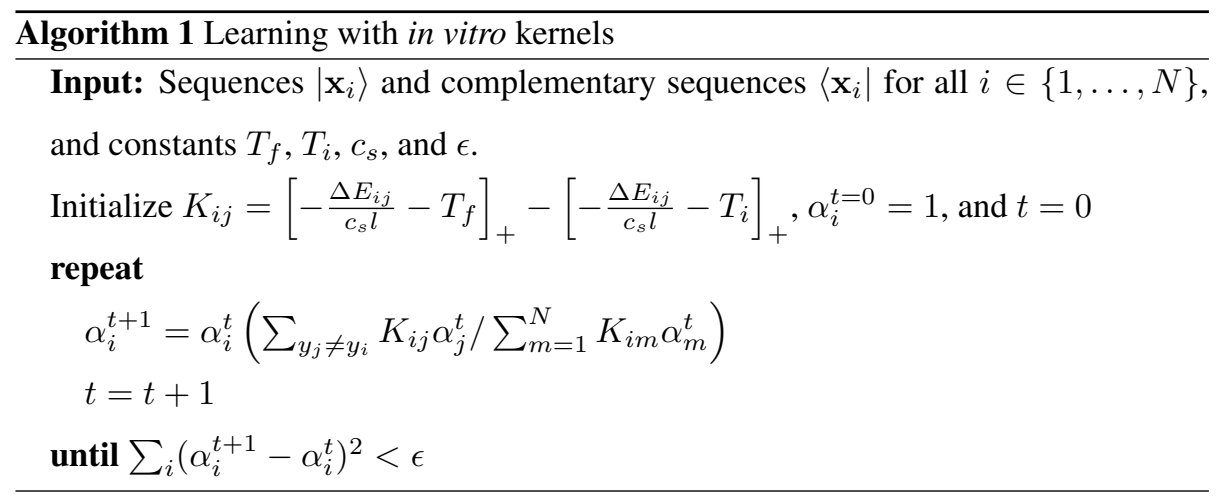

experiment. Data are thus expressed as strings of length 1,000 . We used the kernel defined in Equation (16) using $T_{i}=90^{\circ} \mathrm{C}$ and $T_{f}=20^{\circ} \mathrm{C}$. The results are the average of 5-fold cross-validation. At each validation, 24 different examples are reserved for testing, while the remaining 96 examples are trained.

The other dataset contains DNA sequences that need to be classified according to whether or not they contain a splice region. Three different problems are contained in this set, concerning the classification of expressed sequence tag (EST) in C. elegans, EST in Drosophila, and synthesized sequences, with the details of data collection and processing methods described in [41]. In general, the number of negative samples is larger than positive samples, and we used all the positive data and the same number of random negative data. For the C.elegans set, the 15,507 positive samples are divided into 15 non-overlapping subsets randomly containing 150 training and 700 testing samples. The Drosophila set of 1,583 positive samples is divided into 3 distinct subsets randomly containing 100 training samples and 400 testing samples. The synthetic set has 95 positive training samples and 905 positive testing samples. Among the synthetic sets in [41], we used the sequences where five symbols are randomly replaced.

The classification performance of the algorithm is compared with the conventional SVM and kFDA classifier. The slack variable parameter for the SVM algorithm and the regularization parameter for kFDA are optimized using cross-validation. The ROC curves (Fig. 7) shows that the classification performance of our proposed method is superior to kFDA and performs better than the SVM algorithm on the AML/ALL,

Drosophila, and synthetic sequences. 


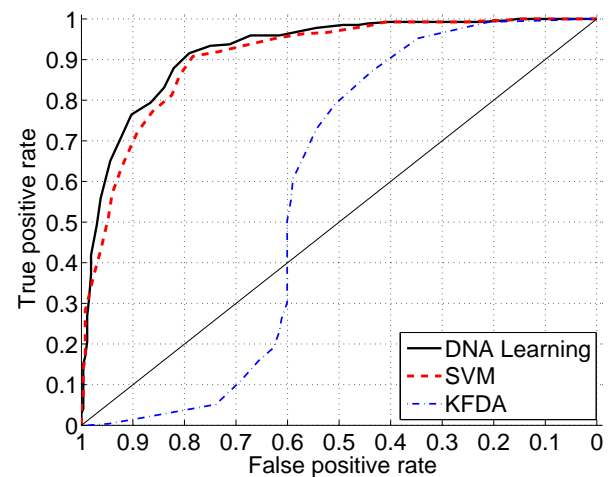

(a)

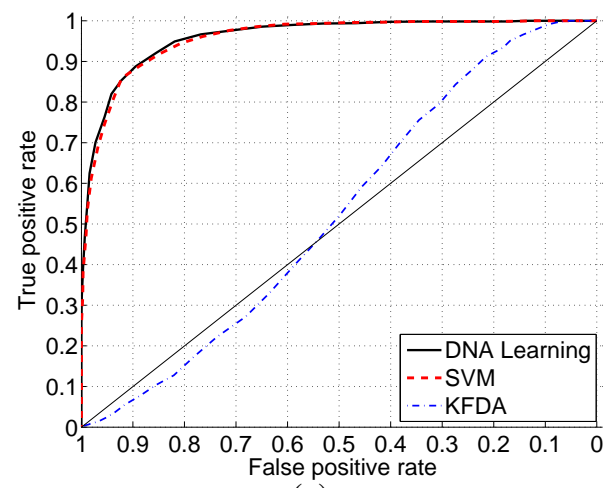

(c)

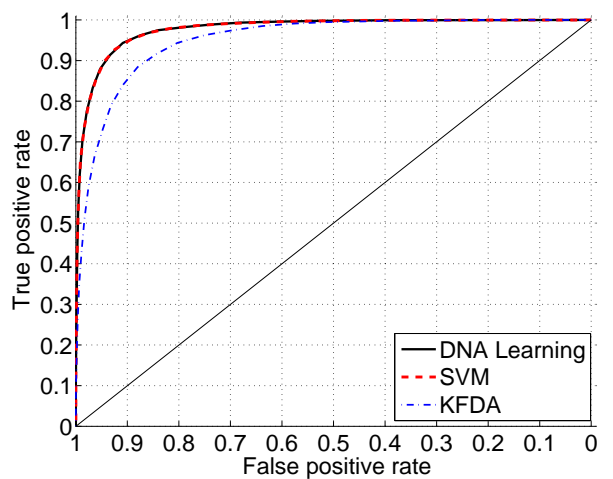

(b)

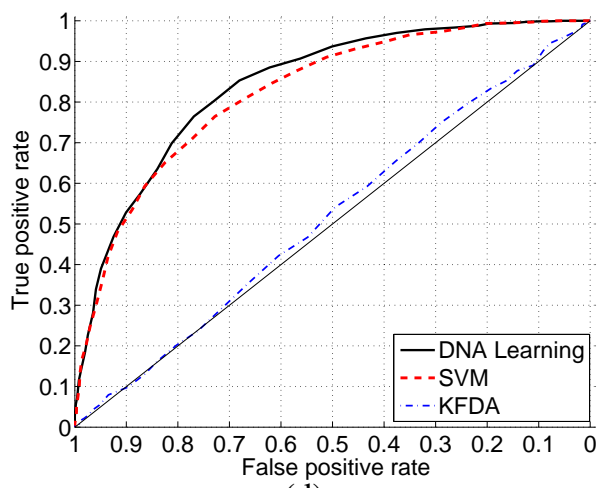

(d)

Figure 7: ROC (Receiver Operating Characteristic) curves. The classification is performed using our DNA learning, SVMs and kernel FDA using the same DNA kernel. The FDA criterion is not in general appropriate in this data. Interestingly, the proposed DNA learning method outperforms or is similar to SVMs in all cases. The slack variable parameter in SVMs and the regularization parameter in FDA are scanned and optimized. In our DNA learning, the algorithm does not have any tunning parameters.

We also present a sample learning curve for the AML/ALL data in Fig. 8 Within a few iterations of the learning algorithm, the test performance increases sharply indicating that the algorithm converges rapidly enough for practical implementation. The resulting embedding in feature space shows that the performance increase is indeed due to increasing the margin of the training data. 


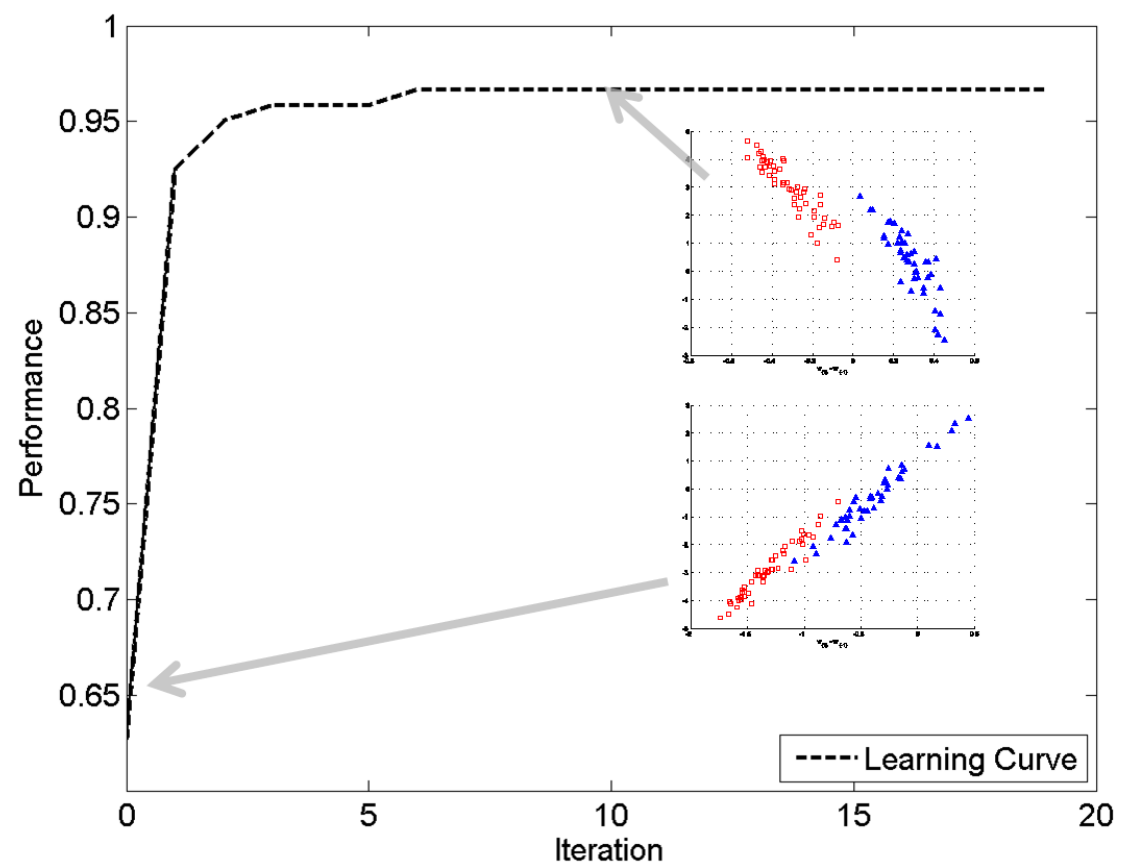

Figure 8: Learning curve (test performance) of AML/ALL data with respect to algorithm iteration and the 2-dimensional embedding of training data on the feature space. Horizontal axis of the insets represents the vector direction $\mathbf{w}_{\{+1\}}-\mathbf{w}_{\{-1\}}$. Vertical axis is the maximum variance direction of the orthogonal space to the discriminating direction.

\section{Biomolecular Implementation}

The implementation of the algorithm on actual biomolecular DNA molecules requires concatenation of various DNA operations. Such operations can include hybridization, denaturing, polymerase chain reaction (PCR, for amplification), and gel electrophoresis (for selection). In this section, we present one example of preliminary implementation using these operations of in vitro classification system. The experiments will show the possibility of implementation in the future, where the actual implementation needs additional complex constraints of DNA operational design. 


\subsection{Implementation procedure}

ity. Second, the common sequence helps make the matrix be positive definite as shown in Theorem 2 and Fig. 5 from the classification accuracy perspective. The dummy code of 9 mer on the 3 ' side of class +1 and 17 mer of the class -1 also can be designed as 
a sticky end, where there is room to design for more selection operations using these dummy sequences.

Table 2: Six different data are represented using twelve kinds of molecules. Each pair $i$ and $i$-C represent one datum containing the data sequence and its complementary sequence.

\begin{tabular}{|c|c|c|}
\hline Class & Index & Sequence \\
\hline $\begin{array}{c}\text { Class }+1 \\
(46 \mathrm{mer} \text { length })\end{array}$ & $\begin{array}{l}1 \\
1-\mathrm{C} \\
2 \\
2-\mathrm{C} \\
3 \\
3-\mathrm{C}\end{array}$ & $\begin{array}{l}\text { 5',AGCAGACTTTAATGTTAATGTTATTATTACTACATCGCACGACTGA3', } \\
\text { 5'CGATGTAGTAATAATAACATTAACATTAAAGTCTGCTCACGACTGA3' } \\
\text { 5'AGCAGACTATGATGTTATTATTATTATTACTACATCGCACGACTGA3', } \\
\text { 5'CGATGTAGTAATAATAATAATAACATCATAGTCTGCTCACGACTGA3' } \\
\text { 5'AGCAGACTATGATGTTATTAATGTTATTACTACATCGCACGACTGA3', } \\
\text { 5'CGATGTAGTAATAACATTAATAACATCATAGTCTGCTCACGACTGA3' }\end{array}$ \\
\hline $\begin{array}{c}\text { Class }-1 \\
\text { (54mer length) }\end{array}$ & $\begin{array}{l}4 \\
4-\mathrm{C} \\
5 \\
5-\mathrm{C} \\
6 \\
6-\mathrm{C}\end{array}$ & 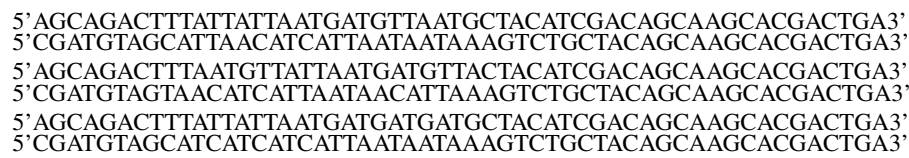 \\
\hline
\end{tabular}

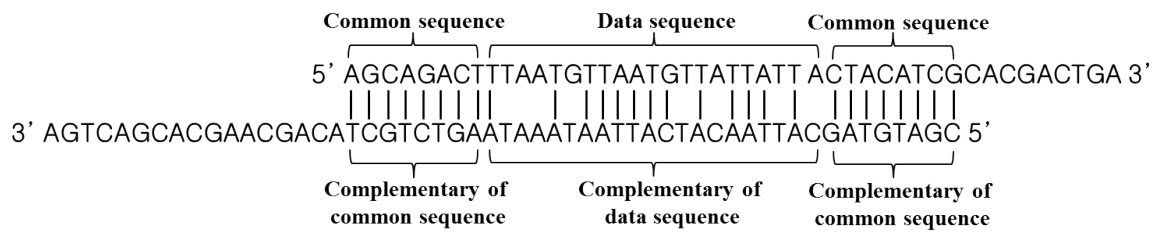

Figure 9: Expected hybridization between molecules 1 and 4-C in Table 2

After enough hybridization, hetero-labeled double strands are selected to update the population as in Theorem 1. In Eq. 6), the total number of double strands containing one particular sequence corresponds to the denominator and the number of heterolabeled double strands among them corresponds to the nominator. Therefore, iterative hetero-labeled selection will lead the distribution having the weight in Theorem 1. After the selection, an amplification process such as PCR can be used to avoid losing the molecules. In this process, we do not have to consider a specific gain, but it is enough to keep the ratio of the population because the classification result is unaffected once the ratio is kept.

In the discriminative phase, when we use the fluorescence intensity of two different binded fluorophores to measure the relative amount of hybridized strands, we 
should make sure that the distance is fixed between the fluorophore and the quencher within a hybridized molecule. Otherwise, the intensity is affected by the distance, and the intensity could not reliably measure the number of double strands. In our method, we considered only the specific hybridization as in Fig.9 9 and tried to disallow any hybridization other than at a pre-determined position by adopting a complementary common sequence throughout all sequences. By allowing only the pre-defined hybridization configurations, we can know in advance the relative fluorescence intensities of different classes when they have the same number of hybridizations, and we can accordingly calibrate the reading of fluorescence intensity of each class.

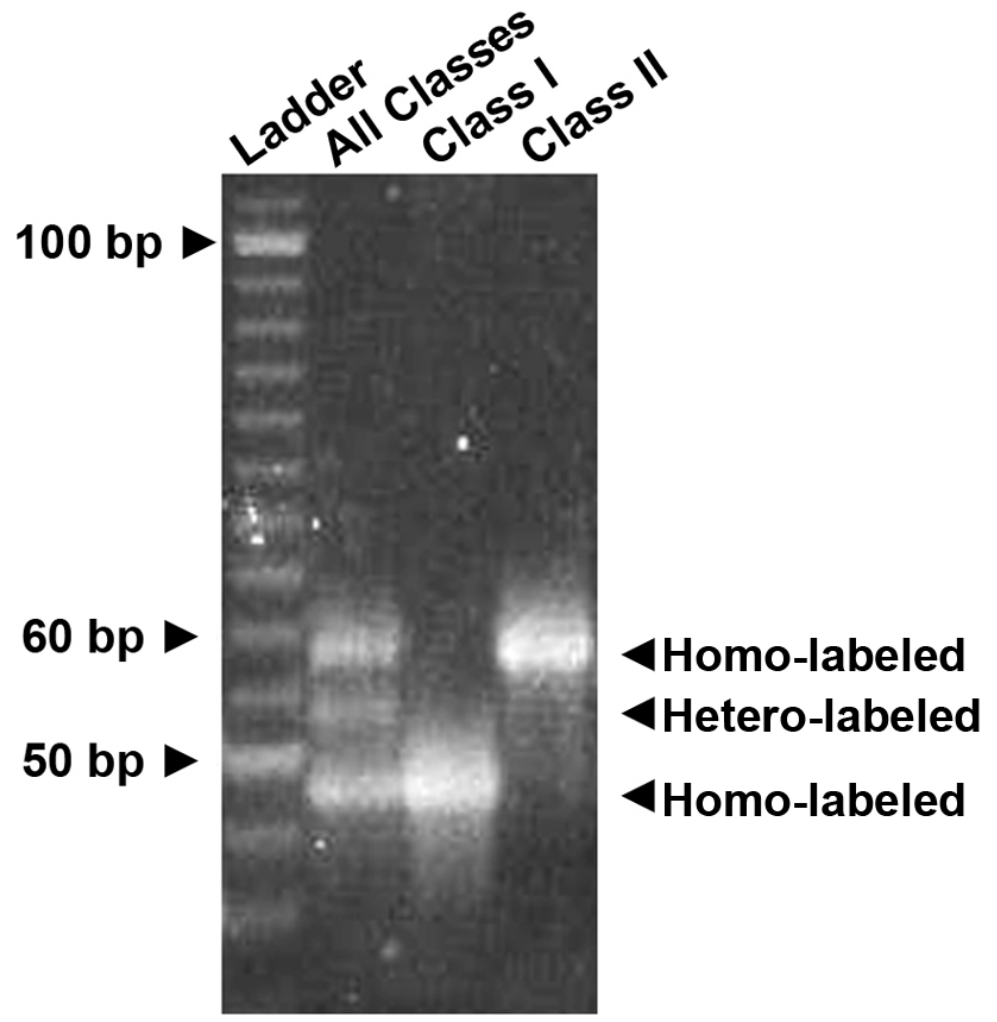

Figure 10: Gel electrophoresis results from mixtures in Table 2 This figure shows the results of three experiments; the first one (second lane) shows the result of all sequence mixtures, and the second and third experiments (third and fourth lanes) are the results of class +1 molecules and class -1 molecules, respectively. 


\subsection{Hybridization implementation}

In Fig. 10, we show the gel electrophoresis result with the hybridized molecules using all sequences presented in Table 2 Here, all species of the oligonucleotide were

mixed with the same concentration in a $1.5 \mathrm{~mL}$ microcentrifuge tube (final $60 \mu \mathrm{M}$ each under $0.5 \mathrm{M} \mathrm{NaCl}$ salt conditions). After heating the mixture at $95^{\circ} \mathrm{C}$ for 3 minutes, we gradually cooled the temperature down in a heat block, after which the tube was incubated overnight with constant rotation $(60 \mathrm{rpm})$ in a $37^{\circ} \mathrm{C}$ incubator. Five microliter products were mixed with $1 \mu \mathrm{l}$ of $6 \mathrm{X}$ loading buffer, and the mixture was loaded into a Spreadex EL300 gel (Elchrom Scientific, Switzerland). Electrophoresis was performed at $120 \mathrm{~V}$ for 3 hours in a SEA 2000 electrophoresis apparatus (Elchrom Scientific). Temperature of the running buffer (TAE) was kept constant at $20^{\circ} C$. The gel was stained with SYBR Gold, and visualized using Bio-Rad gel doc 2000 (Bio-Rad, USA).

525 We also estimated the quantity of molecules in the three bands. The average and standard deviation for 15 trials are presented in Table. 3 . As expected, when we consider the resulting $2 \times 2$ matrix representing the amount of double strands of four possible class combinations of the primary and complementary strands, the matrix for this experiment is positive definite as a necessary condition for the positive definiteness of the kernel.

Table 3: Hybridized amount (IDV)

\begin{tabular}{|l|l|}
\hline Homo (class +1) & $209.2 \pm 13.8$ \\
\hline Hetero & $200.5 \pm 15.1$ \\
\hline Homo (class -1) & $206.4 \pm 15.0$ \\
\hline
\end{tabular}

Another expectation is the linear relationship of the amount with respect to the binding energy. In Section 4 we explained that the distribution of the differently hybridized molecules will not follow the Boltzmann distribution with many molecules. According to our kinetic model, the amount of molecules will be proportional to the 
energy at first, and the distribution will stay in one nearby local attractor distribution at equilibrium. Here, the equilibrium distribution can be any attractor making all equations on the right hand side in Eq. (18) be zero. Therefore, the final distribution is close to linear rather than exponential which is the case when the population follows a Boltzmann distribution.

\section{Conclusions}

We introduced a biomolecular algorithm for learning with DNA moleculesInterpreted within the context of kernel machines, the resulting algorithm can be viewed as learning a weight vector associated with the convex cones of the data in the associated feature space. Simulations show that the proposed molecular learning algorithm is competitive with state-of-the-art machine learning algorithms.

We also showed the possibility of exploiting the algorithm to real in vitro implementation. In particular, the thermodynamic simulation of constructing kernel matrix shows that a simple cooling schedule of hybridization guarantees the positive definiteness of the resulting matrix. Given the promising nature of these results, we hope that experimental groups will consider additional experiments building upon our proposed molecular algorithm and analysis. Future work needs to consider how to optimize yield in such biomolecular processes. We hope that with such work, learning and classification will be implemented seamlessly in a living cell.

\section{Acknowledgements}

This work was partly supported by AFOSR (FA2386-12-1-4087, FA9550-15-10002), NRF (NRF-2010-0017734-Videome), DAPA, ADD, and BK21Plus.

\section{References}

[1] Alleyne, T.-M., Peña-Castillo, L., Badis, G., Talukder, S., Berger, M.-F., Gehrke, A.-R., Philippakis, A.-A., Bulyk, M.-L., Morris, Q.-D., \& Hughes, T.-R. (2009). Predicting the binding preference of transcription factors to individual DNA kmers. Bioinformatics, 25, $1012-1018$. 
[2] Andronescu, M., Fejes, A. P., Hutter, F., Hoos, H. H., \& Condon, A. (2004). A new algorithm for RNA secondary structure design. Journal of Molecular Biology, 336, 607 - 624 .

[3] Ben-Hur, A., Ong, C.-S., Sonnenburg, S., Schölkopf, B., \& Rätsch, G. (2008). Support vector machines and kernels for computational biology. PLoS Computational Biology, 4, e1000173.

[4] Benenson, Y. (2012). Biomolecular computing systems: principles, progress and potential. Nature Reviews Genetics, 13, 455 - 468.

[5] Bennett, C.-H. (1982). The thermodynamics of computation - a review. International Journal of Theoretical Physics, 21, 905 - 940.

[6] Britten, R. J., \& Kohne, D. E. (1968). Repeated Sequences in DNA. Science, $161,529-540$.

[7] Burges, C. (1998). A Tutorial on Support Vector Machines for Pattern Recognition. Data Mining and Knowledge Discovery, 2, 121 - 167.

[8] Cheok, M.-H., Yang, W., Pui, C.-H., Downing, J.-R., Cheng, C., Naeve, C.W., Relling, M.-V., \& Evans, W.-E. (2003). Treatment-specific changes in gene expression discriminate in vivo drug response in human leukemia cells. Nature Genetics, 34, $85-90$.

[9] Davis, L., Hawkins, J., Maetschke, S. R., \& Boden, M. (2006). Comparing SVM Sequence Kernels: A Subcellular Localization Theme. 2006 Workshop on Intelligent Systems for Bioinformatics (WISB 2006), 73, 39 - 47.

[10] Dirks, R. M., Bois, J. S., Schaeffer, J. M., Winfree, E., \& Pierce, N. A. (2007). Thermodynamic Analysis of Interacting Nucleic Acid Strands. SIAM Review, $49-1,65-88$.

[11] Gärtner, Thomas (2003). A survey of kernels for structured data. SIGKDD Explorations. 
[12] Gärtner, T., Lloyd, J.-W., \& Flach, P.-A. (2004). Kernels and distances for structured data. Machine Learning, 57, 205 - 232.

[13] Gillespie, D. (1977). Exact stochastic simulation of coupled chemical reactions. The Journal of Physical Chemistry, 81, 2340 - 2361.

[14] Gillespie, D. (2007). Stochastic simulation of chemical kinetics. Annual Review of Physical Chemistry, 58, $35-55$.

[15] Haasdonk, B. (2005). Feature space interpretation of SVMs with indefinite kernels. IEEE Transactions on Pattern Analysis and Machine Intelligence, 27, 482 -492 .

[16] Hannenhalli, S. (2008). Eukaryotic transcription factor binding sites-modeling and integrative search methods. Bioinformatics, 24, 1325 - 1331.

[17] Hjelmfelt, A., Weinberger, E. D., \& Ross, J. (1991). Chemical implementation of neural networks and Turing machines. Proceedings of the National Academy of Sciences USA, 88, 10983 - 10987.

[18] Hopfield, J. (1982). Neural networks and physical systems with emergent collective computational abilities. Proceedings of the National Academy of Sciences, $79,2554-2558$.

[19] Kahan, M., Gil, B., Adar, R., \& Shapiro, E. (2008). Towards molecular computers that operate in a biological environment. Physica D: Nonlinear Phenomena, $9,1165-1172$.

[20] Kim, J., Hopfield, J., \& Winfree, E. (2005). Neural network computation by in vitro transcriptional circuits. Advances in Neural Information Processing Systems $17,681-688$.

[21] Kim, J., White, K. S., \& Winfree, E. (2006). Construction of an in vitro bistable circuit from synthetic transcriptional switches. Molecular Systems Biology, 2, 1 -12 . 
[22] Kim, J.-S., Lee, J.-W., Noh, Y.-K., Park, J.-Y., Lee, D.-Y., Yang, K.-A., Chai, Y.G., Kim, J.-C., \& Zhang, B.-T. (2008). An evolutionary Monte Carlo algorithm for predicting DNA hybridization. BioSystems, 91, $69-75$.

[23] Kowalczyk, A. (2000). Maximal margin perceptron. Advances in Large Margin Classifiers, 75 - 113, MIT Press, Cambridge, MA, USA.

[24] Laplante, J.-P., Pemberton, M., Hjelmfelt, A., \& Ross, J. (1995). Experiments on Pattern Recognition by Chemical Kinetics. Journal of Physical Chemistry, $9910063-10065$.

[25] Leslie, C., Eskin, E., \& Noble, W. S. (2002). The spectrum kernel: a string kernel for SVM protein classification. Pacific Symposium on Biocomputing, 7, $564-575$.

[26] Leslie, C., Kuang, R., \& Eskin, E. (2003). Inexact matching string kernels for protein classifcation. Kernel Methods in Computational Biology, 95 - 112. MIT Press.

[27] Lim, H.-W., Lee, S.-H., Yang, K.-A., Lee, J.-Y., Yoo, S.-I., Park, T.-H., \& Zhang, B.-T. (2010). In vitro molecular pattern classification via DNA-based weightedsum operation. BioSystems, 100, $1-7$.

[28] Lo, J. (2011). A low-order model of biological neural networks. Neural Computation, 23, $2626-2682$.

[29] Lu, J., Getz, G., Miska, E.-A., Alvarez-Saavedra, E., Lamb, J., Peck, D., SweetCordero, A., Ebert, B.-L., Mak, R.-H., Ferrando, A.-A., Downing, J.-R., Jacks, T., Horvitz, H.-R., \& Golub, T.-R. (2005). MicroRNA expression profiles classify human cancers. Nature, 435, $834-838$.

[30] Lu, Y., Sato, Y., \& Amari, S.-I. (2011). Traveling bumps and their collisions in a two-dimensional neural field. Neural Computation, 23, 1248 - 1260.

[31] Lu, Z., Leen, T., \& Kaye, J. (2011). Kernels for longitudinal data with variable sequence length and sampling intervals. Neural Computation, 23, 2390 - 2420. 
[32] Middendorf, M., Kundaje, A., Wiggins, C., Freund, Y., \& Leslie, C. (2004). Predicting genetic regulatory response using classification. ISMB/ECCB (Supplement of Bioinformatics), $232-240$.

[33] Mika, S., Ratsch, G., Weston, J., Scholkopf, B., \& Muller, K.-R. (1999). Fisher discriminant analysis with kernels. Neural Networks for Signal Processing, volume IX, $41-48$.

[34] Mills Jr., A. P. (2002). Gene expression profiling diagnosis through DNA molecular computation. Trends in Biotechnology, 20, $137-140$.

[35] Mills Jr., A. P., Turberfield, M., Turberfield, A. J., Yurke, B., \& Platzman, P. M. (2001). Experimental aspects of DNA neural network computing. Soft Computation, $5,10-18$.

[36] Mir, K. (1999). A restricted genetic alphabet for DNA computing. In DNA Based Computers II, 44, 243 - 246.

[37] Ong, C., Mary, X., Canu, S., \& Smola, A. (2004). Learning with non-positive kernels. International Conference on Machine Learning, 639 - 646.

[38] Poje, J. E., Kastratovic, T., Macdonald, A. R., Guillermo, A. C., Troetti, S. E., Jabado, O. J., Fanning, M. L., Stefanovic, D., and Macdonald, J. (2014). Visual display that directly interface and provide read-outs of molecular states via molecular graphics processing units. Angewandte Chemie International Edition, $53,9222-9225$.

[39] Qian, L., Winfree, E., \& Bruk, J. (2011). Neural network computation with DNA strand displacement cascades. Nature, 475, $368-372$.

[40] Rätsch, G. \& Sonnenburg, S. (2004). Accurate splice site detection for caenorhabditis elegans, 277 - 298. MIT Press series on Computational Molecular Biology. MIT Press.

[41] Rätsch, G., Sonnenburg, S., \& Schäfer, C. (2006). Learning interpretable SVMs for biological sequence classification. BMC Bioinformatics, 7(Suppl. 1):S9. 
[42] Rieck, K. \& Laskov, P. (2008). Linear-time computation of similarity measures for sequential data. Journal of Machine Learning Research, 9, 23 - 48.

[50] van't Veer, L.-J. \& Bernards, R. (2008). Enabling personalized cancer medicine through analysis of gene-expression patterns. Nature, 452, $564-570$.

[51] Vert, J.-P., Thurman, R., \& Noble, W.-S. (2006). Kernels for gene regulatory regions. Advances in Neural Information Processing Systems 18, 1401 - 1408. 
[52] Zadeh, J. N. and Steenberg, C. D. and Bois, J. S. and Wolfe, B. R. and Pierce, M. B. and Khan, A. R. and Dirks, R. M. and Pierce, N. A. (2011). NUPACK: Analysis and design of nucleic acid systems. Journal of Computational Chemistry, $32,170-173$.

[53] Zhang, B.-T. \& Jang H.-Y. (2005). A Bayesian algorithm for in vitro molecular evolution of pattern classifiers. Proc. 2004 International Annual Meeting on DNA Computing (DNA 10), LNCS, 3384, 458 - 467.

[54] Zhang, B.-T. (2008). Hypernetworks: A molecular evolutionary architecture for cognitive learning and memory. IEEE Computational Intelligence Magazine, 3 , $49-63$.

[55] Zhang, D., Turberfield, A., Yurke, B., \& Winfree, E. (2007). Engineering entropy-driven reactions and networks catalyzed by DNA. Science, 318, 1121 1125 .

[56] Zien, A., Rätsch, G., Mika, S., Schölkopf, B., Lengauer, T. \& Müller, K.-R. (2000). Engineering support vector machine kernels that recognize translation initiation sites. Bioinformatics, 16, $799-807$.

[57] Zwillinger, D. (1996). CRC standard mathematical tables and formulae. CRC Press. 